eintreten oder dass die entsprechenden Stellen der anderen Hemisphäre die ausgefallenen Functionen übernehmen sollen.

Wir haben aus unserem grösseren Material absichtlich nur diese wenigen Fälle ausgelesen und z. B. die Fälle von Hirntumoren bei Seite gelassen, wo wegen der begleitenden Störungen der Intelligenz, des Gedächtnisses u. s. w., es oft schwierig ist $\mathrm{zu}$ entscheiden, ob richtige Aphasie vorhanden war oder nicht, und sprechen den Wunsch aus, dass noch möglichst viele genau beobachtete Aphasiefälle mitgetheilt werden mögen, damit ein späterer Autor, wenn Bausteine genug vorliegen, sich an's Aufbauen des Eingangs erwähnten Gebäudes machen könne.

Zum Schlusse ist es mir eine angenehme Pflicht, meinem hochverehrten Chef, Herrn Professor Eichhorst, für die gütige Ueberlassung der Krankengeschichten und für das rege Interesse an dieser Arbeit meinen verbindlichsten Dank auszusprechen.

\title{
XVI.
}

\section{Zur Aetiologie des primären Carcinoms der Gallenblase.}

\author{
Von Dr. F. Siegert, \\ Assistenten am pathologischen Institut zu Genf.
}

Ueber den primären Krebs der Gallenblase liegt eine grosse Anzahl von Abhandlungen vor. So haben in den letzten $25 \mathrm{Jah}$ ren die Dissertationen von Bertrand ${ }^{1}$, Behrens ${ }^{2}$, Kohn ${ }^{3}$, Kraus $^{4}$, H. Zenker ${ }^{5}$, Chachamovicz ${ }^{6}$, Morin ${ }^{7}$, Peters ${ }^{8}$, Martins ${ }^{9}$, Thiedemann ${ }^{10}$ und Ohloff ${ }^{11}$ denselben zum Gegenstande in klinischer und anatomischer Beziehung, und eine grosse Reihe von Einzelfällen sind in den verschiedenen Fachschriften besprochen. Besonders eine Frage wird in allen diesen Abhandlungen auf's Neue erörtert: Ist der Krebs der Gallenblase die Ursache oder im Gegentheil die Folge der so häufig gleichzeitig beobachteten Cholelithiasis? Beide Ansichten finden ihre 
Vertreter. Während die Kliniker und ihre Schüler zu der Anschauung neigen, dass die durch den Krebs bedingten Veränderungen der Gallenblase, wie Behinderung in deren Contraction, anomale Secretion oder Bildung von freien Körpern durch Zerfall des Tumors eine nachträgliche Entstehung von Gallensteinen zur Folge haben, sehen die patholog. Anatomen und ihre Schüler in der Cholelithiasis die Ursache des Carcinoms, sie erklären seine Aetiologie durch die Wirkung eines chronischen Reizes im Sinne Virchow's.

In diesem Sinne spricht sich Klebs ${ }^{12}$ schon sehr entschieden aus, und Prof. Zahn stellte in dem unter No. 11 später erwähnten Falle von primärem Krebs des Pankreas mit Metastasen in der Gallenblase die Diagnose: „secundärer Krebs der Gallenblase" schon aus dem Fehlen von Gallensteinen, - eine Diagnose, die sich durch den weiteren Verlauf der Section, sowie die genauere Untersuchung bestätigte.

Zwei Fälle von primärem Caroinom der Gallenblase, die ich hier innerhalb weniger Tage zu beobachten Gelegenheit hatte, veranlassten mich, diesem Gegenstand auf's Neue näher zu treten, wobei mir 5 weitere Fälle aus der hiesigen, von Prof. Zahn geschaffenen Sammlung zu Statten kamen. Die 5 ersten der in Folgendem kurz angegebenen Fälle betreffen in Alkohol gut gehärtete Präparate; Fall 6 und 7 secirte ich selbst und untersuchte sie in frischem Zustande.

1. Frau B., 76 Jahre alt. 1880. No. 153.

Gallenblase mit der Leber fest verwachsen, sehr stark erweitert, faustgross. Wand verdickt, in einen derben Tumor verwandelt, durch den der erweiterte Ductus cysticus hindurch zieht. Schleimhaut böckerig, zum Theil ulcerirt. Inhalt: schleimige, gelbe Galle und viele erbsengrosse, facettirte Gallensteine. Ductus cysticus in den Tumor eingebettet, Ductus choledochus verengert, Wandung verdickt, krebsig infiltrirt. Ductus hepaticns und seine Zweige erweitert, Wand unverändert. Metastasen in der Leber, im Kopf des Pankreas ein Knoten, Lymphknoten um die Pfortader krebsig infiltrirt und vergrössert.

Mikroskopischer Befund: Schleimhaut wie Submucosa sehr verdickt, in skleröses Bindegewebe verwandelt, in dem communicirende Stränge von kleinen, polygonalen Zellen ohne Intercellularsubstanz eingebettet sind. Gegen die Serosa hin sind die Alveolen nur durch in länglich-ovalen Beerden angeordnete Pigment- und Fettkörnchen angedeutet. Gefässwände sehr verdickt. 
2. 1888. No. 189. Fran E., 57 Jahre alt.

Gallenblase mit der Leber fest verwachsen, mässig erweitert, Wandung verdickt, derbe. Schleimhaut in grosser Ausdehnung ulcerirt. Inhalt: eingedickte Galle und mehrere grosse Gallensteine. Ductus cysticus und die übrigen Gallengänge unverändert. Metastasen in der Leber, Lymphknoten im Leberbilus und um den Pankreaskopf vergrössert, krebsig infiltrirt.

Mikroskopischer Befund: wie in Fall 1.

3. 1890. No. 83. Frau B., 76 Jahre alt.

Gallenblase geschrumpft, mit Leber, Duodenum und Netz fest verwachsen. Wand stark verdickt, Schleimhaut ulcerirt. Inhalt: einige grosse, würfelförmige Gallensteine. Metastasen in Leber und Peritonänm. Lymphknoten des Leberhilus krebsig infiltrirt.

Mikroskopischer Befund: Wand gleichmässig verdickt, Bindegewebe sklerotisch, Gefässwände verdickt. Schleimhautfalten zottenförmig bypertrophirt, ulcerirt, von Mikrokokken bedeckt und durchsetzt. Alveolen von der Oberfläcbe nach der Tífe an Grösse abnehmend, erfüllt von kleinen polygonalen Zellen mit grossem Kern.

4. 1890. No. 61. Frau Ch., 70 Jahre alt.

Gallenblase erweitert, stark birnengross, mit Netz und Quercolon verwachsen. Wandung sehr verdickt, Hals bis zur Mündung des Ductus cysticus ringförmig ulcerirt, Schleimhaut von blumenkohlähnlichem Aussehen, unregelmässig zottig. Ductus cysticus für den kleinen Finger durchgängig, sehr verdickt, Schleimhaut in gleicher Weise nlcerirt. Ductus choledochus ebenfalls erweitert und verdickt, Schleimhaut nicht ulcerirt. Ductus bepaticus und seine Verzweigungen dilatirt, Wandung nicht verändert.

Inbalt der Gallenblase: etwa $180 \mathrm{ccm}$ schleimiger, blasser Galle und viele facettirte Gallensteine. Metastatische Knoten in der Leber und einer im Pankreaskopf. Lymphknoten des Leberhilus und in der Umgebung des Pankreaskopfes sowie im Netz verdickt, krebsig infiltrirt.

Mikroskopischer Befund: Schleimhaut des Fundus der Gallenblase unverändert. Schleimbant des Blasenbalses von Epithel entblösst, ulcerirt, von Mikrokokken bedeckt. Schleimhautfalten hier papillenartig gewuchert und verdickt, zum Theil durch Ulceration geschwunden. In der Basis der Papillen finden sich grosse communicirende Alveolen, erfüllt von kleinen, polygonalen Zellen mit grossem Kern und schmalem, körnigem Protoplasma, ohne Intercellularsubstanz. In der Alveolenwand bypertrophische Bündel glatter Musculatur. In den tieferen Schichten erscheinen die Alveolen schmäler, die Zellen fettig entartet, schwer zu färben. In der Submucosa, die in derbes Bindegewebe verwandelt ist, sind die einstigen Alveolen durch Gruppen von Fettkörnchen angedeutet. Serosa verdickt, sehnig glänzend.

5. Frau K., 60 Jahre alt. 1891. No. 149.

Gallenblase von normaler Grösse und stark verdickter Wandung. Schleimhaut ulcerirt, höckerig verdickt, ron drüsigem Aussehen. Inbalt: schleimige 
mit Blut und Blutgerinnseln unterinischte Galle und ein taubeneigrosser Cholestearinstein. Ductus hepaticus und Lymphknoten am Gallenblasenhals ergriffen. Metastasen in der Leber und rechten Lunge.

Mikroskopischer Befund: ganz wie Fall No. 3.

6. G., Mann von 65 Jahren. 1892. No. 164.

Leber oben mit $\mathrm{Zwerehfell}$ und am unteren Rand mit Pylorus und Colon ascendens verwachsen. Beim Aufbeben des Quercolons und Duodenums, die gleichfalls adbärent sind, entsteht in der Gegend der Gallenblase eine Oeffnung, aus der eitrige Flüssigkeit und viele erbsengrosse facettirte Steine hervorquellen. Die Oeffuung entstand durch Trennung des Duodenums von der ganz geschrumpften und ulcerirten Gallenblase. Gallenblase, Ductus cysticus und hepaticus erfüllt von über 100 kirschkerngrossen Gallensteinen. Ductus cysticus durch Ulceration sebr erweitert, für einen Finger durchgängig. Ductus choledochus enge, undurchgängig. Faustgrosser Tumor im rechten Leberlappen an der hinteren Fläcbe, mit grosser, strabliger Narbe im Centrum, viele kleinere Tumoren an der Oberfläche mit centraler Depression, andere in der Tiefe. Vena portarum und deren Verzweigungen erfüllt von einem verzweigten Thrombus von gelbröthlicher Farbe, gleichmässig weicher Consistenz. Dieser Thrombus sitzt der Venenwand nur fest auf an der Verwachsungsstelle des in den Tumor aufgegangenen Gallenblasenhalses mit dem rechten Pfortaderaste.

Mikroskopischer Befund: Auf Querschnitten des noch erhaltenen Restes der Wand der sehr geschrumpften Gallenblase erscheint die Schleimhaut ulcerirt, von Mikrokokhen bedeckt. Die sehr verdickte Submucosa zeigt spärliche, der Wand parallele, dureb Querzïge verbundene Epithelstränge, die in jhrer Anordnung sich leicht als die von Epithelien erfüllten Lymphgefässe erkennen lassen. Der Inhalt derselben besteht aus kleinen polygonalen Zellen mit grossem Kern, ohne Intercellularsubstanz; die Alveolenwand aus sklerösem, sehnig glänzendem Bindegewebe. Die Arterienwände sind stark verdickt, das Lumen einzelner ist obliterirt. Schleimhautzotten und glatte Musculatur sind durch Ulceration geschwinden. Die Structur des Tumors ist eine deutliche in den kleinen metastatischen Lebertumoren. Die an der Peripherie von leicht zu färbenden Zellen erfüllten, schmalen Alveolen sind näher dem Centrum des Tumors gross und mit fettig degenerirtem Zellmaterial erfüllt. Im Centrum selbst findet sich sklerotisches Bindegewebe in strabliger Anordnung.

Der verzweigte Thrombus der Pfortader besteht aus spärlichem Bindegewebe und Fibrin, in Maschen angeordnet, $z$ wischen denen sich das gleiche Zellmaterial findet, wie in den beschriebenen Tumoren. Statt der Gefässe enthält der Thrombus unregelmässige Spalten und von Blut erfüllte Räume.

An seiner Oberfläche findet sich wellig angeordnetes Fibrin mit wenigen Leukocyten.

7. Frau J., 74 Jahre alt. 1892. No. 168.

Leber und Gallenblase änsserlich normal. Bei der Eröffnung der Gallen- 
blase finden sich in ihr neben reichlicher grüner Galle 6 erbsen- bis klein kirschgrosse facettirte Gallensteine im Fundus eingeklemmt. Die im übrigen normale Gallenblasenwand erscheint am Fundus in der Ausdehnung einer Linse leicht verdickt, die Schleimhautfalten etwas vergrössert. Der Anwesenheit der Steine wegen wird die genauere Untersuchung beschlossen, welche folgenden Befund ergiebt.

Die. Gallenblase ist weder vergrössert, noch verdickt, mit Ausnahme der erwähnten Stelle am Fundus. Die Schleimbaut erscheint an dieser letzteren papillenartig gewuchert und bängt mit der Submucosa fest zusammen. Der Peritonäalüberzug zeigt keine makroskopische Veränderung.

Ein Stück aus dem Blasenbals, sowie die verdickte Stelle werden in conc. Alkohol gehärtet, in Celloidin eingebettet und mit dem Mikrotom geschnitten.

Das dem Blasenbals entnommene, makroskopisch unveränderte Stück der Gallenblase zeigt auch mikroskopisch keine Veränderung. Das hohe, durch die Galle grüngefärbte Cylinderepithel mit Cuticularsaum ist stellenweise erhalten und bildet einen einschichtigen Belag der Membrana propria. Diese begleitet bald die charakteristischen Erhebungen der Schleimbaut in Zottenform, bald kleine Einsenkungen unter das Niveau der Schleimhant, welche weder verdickt noch sonst entzündlich verändert ist. Feinfaseriges, zartes Bindegewebe heftet sie an die aus derbem, dichtem Bindegewebe bestehende Submucosa, deren Blutgefässe sich völlig normal verhalten. Auch das die Submucosa überziehende Peritonäum ist unverändert.

Ganz anders liegen die Verhältnisse in der dem Fundus entnommenen, makroskopisch nur verdickten Stelle.

Der Epithelbelag an der Oberfläche fehlt hier rollkommen. Lange verzweigte Papillen erheben sich aus der Mucosa dicht bei einander stehend über die_Oberfläche. Dieselben sind zellig infiltrirt, die glatten Muskelfaserbündel sind hypertrophisch, die Gefässe haben eine verdickte Wand und ein verengertes Lumen. Zwischen diesen zapfenförmigen Schleimhautpapillen finden sich die tiefen Buchten ausgekleidet mit hohem Cylinderepithel, das vielfach in ganzen Reihen abgestossen ist. Indem solche Buchten auf dem Quer-, Längs- und Schrägschnitt getroffen erseheinen, konnten sie bei flüchtiger Betrachtung den Eindruck des Cylinderzellenkrebses machen. Doch liegen diese seheinbaren, übrigens nicht mit einander communicirenden Alveolen alle in der Mucosa und sind von dicken Bündeln glatter Muskelfasern umgeben. Die Blutgefässe zeigen eine sebr verdickte Wandung und sind zum Theil durch Endarteriitis obliterans völlig undurehgängig geworden. In den tiefsten Schichten der Mucosa, reichlicher aber in der mit ihr durch dichtes, derbes Bindegewebe fest verbundenen Submucosa finden sich zahlreiche, mit einander communicirende Alveolen mit bindegewebiger, zum Theil zellig infiltrirter Wand, erfüllt von kleinen, polygonalen Zellen mit grossem Kern und schmalem Protoplasmasaum. Intercellularsubstanz fehlt, die Alveolen sind theils völlig ausgefüllt von diesen epithelartigen Zellen, 
theils bilden diese einen ein- oder mehrschichtigen Belag. Nirgends lässt sich ein Zusammenhang dieser Alveolen mit der Oberfläche der Schleimbaut nachweisen, nirgends ein Uebergang der in den tiefen Buchten reichlich erbaltenen bohen Cylinderepithelien in die kleinen, polygonalen Zellen der Alveolen. Auch die Färbung unterscheidet diese von jenen. Wäbrend der Alveolarinhalt mit Hämatoxylin und Pikrocarmin eine gute Kernfärbung zeigt, bei fast ungefärbtem Protoplasma, gelingt die Färbung der hohen Cylinderepitbelien der Gallenblase nur unvollständig, ihr Protoplasma erscheint punktförmig getrübt, der Kern färbt sich sebr undeutlich, oft gar nicht.

Der Peritonäalüberzug zeigt ausser der Verdickung der Gefässwände keine besondere Veränderung.

Wir haben es demnach hier zu thun mit einem Fall eines primären Carcinoms der Gallenblase in seinen ersten Anfängen. Die Geschwulst ist etwa einen Millimeter dick und von der Grösse einer Linse. Serosa und obere Schicht der Schleimhaut sind von dem Tumor nicht ergriffen, zeigen hingegen chronisch entzündliche Veränderungen.

Die in Vorstehendem besprochenen 7 Fälle von primärem Krebs der Gallenblase zeigen, wie wir gesehen, ausnahmslos Gallensteine als Inhalt der Gallenblase. Ihre Anwesenheit in Fall 7 lässt es als wahrscheinlich annehmen, dass sie der Entwickelung des Carcinoms vorausgingen. Denn einerseits ist die anatomische Veränderung der Gallenblase eine noch sehr geringe und aus ihr lässt sich ein Grund zur Bildung von Gallensteinen nicht herleiten. Andererseits aber sprechen die neben dem Carcinom, und zwar in grösserer Ausdehnung vorhandenen entzündlichen Veränderungen für die Ansicht, dass hier die im Fundus der Gallenblase eingeklemmten Steine einen chronischen Reiz ausübten. Klinische Thatsachen lassen uns im Stich, weil Symptome eines Gallenblasenleidens nicht vorhanden waren und auch in den übrigen 6 Fällen war klinisch über das Vorausgehen oder Nachfolgen der Cholelithiasis nichts Sicheres zu ermitteln.

Der Umstand aber, dass in allen 7 Fällen Cholelithiasis vorhanden war, dass ferner auch in den von der Neubildung nicht ergriffenen Theilen der Gallenblase die Zeichen einer chronischen Entzündung: Neubildung von Bindegewebe und Verdickung der Gefässwände zu beobachten sind, lässt uns die Wirkung eines chronischen Reizes im Sinne Virchow's mehr als wahrscheinlich erscheinen und nöthigt uns zu der Frage: 
Ist der Krebs der Gallenblase eine Folge vorausgegangener Cholelithiasis?

Zwei Arten der Beweisführung finden wir zur Beantwortung dieser Frage in bejahendem Sinne immer wieder angewendet. Einmal wird versucht, an der Hand klinischer Beobachtungen zu beweisen, dass zunächst die sicheren Zeichen einer Cholelithiasis vorhanden waren, und dass erst später die Erscheinungen eines allmählich wachsenden Tumors der Gallenblasenwand hinzutraten. Andererseits wird durch die anatomischen Thatsachen bewiesen, dass in diesem oder jenem Falle die Grösse der vorhandenen Gallensteine in keinem Verhältniss zu Alter oder Umfang des Carcinoms steht. H. Zenker glaubt durch die Untersuchung von Gallenblasennarben zum Ziel zu gelangen, von der Anschauung ausgehend, dass die in solchen beobachtete Wucherung der Schleimdrüsen, ähnlich wie in den Narben der runden Magengeschwüre, zar Bildung des Carcinoms führen könne.

Eine dritte Art der Beweisführung vermisse ich in der Literatur, obwohl sie mir einfacher und sicherer erscheint, als die bisher versuchten. Gehen wir einmal von der gegentheiligen Ansicht aus, dass nehmlich der Gallenblasenkrebs die Cholelithiasis verursacht, nicht umgekehrt. Dann müssten sich Gallensteine in gleicher Häufigkeit finden beim primären wie secundären Gallenblasenkrebs.

Es wäre demnach zu untersuchen: 1. In wie viel Fällen von primärem Gallenblasenkrebs finden sich Gallensteine? 2. Wie verhalten sich in dieser Beziehung die secundären, metastatischen Gallenblasenkrebse?

Die Beantwortung der ersten Frage ergiebt sich durch eine Zusammenstellung der bisher veröffentlichten Fälle von primärem Krebs der Gallenblase. Doch lassen sich hier nur diejenigen Fälle verwerthen, von denen sich mit Sicherheit beweisen lässt, dass sie primären Krebs der Gallenblase, nicht der Gallenwege im Allgemeinen betreffen, und in denen der Inhalt der Gallenblase angegeben ist. Dieser Grund nöthigt mich, auf einzelne Fälle näher einzugehen und andererseits die im Anhang an meine Tabelle erwähnten Fälle unberücksichtigt za lassen, 
Tabelle von H. Zenker (a. a. 0.). $\begin{array}{lllllllll}53 & 6 & 39 & 8 & 41 & 5 & 7\end{array}$

Lacaze-Duthiers, Bull. de la société

anatom. 1847. p. 335 . . . . . . Rippol, ibidem. 1849. p. 359 . . . Icery, ibidem. 1853. p. 75 .. . .

Mahieux, ibidem. 1853. p. 180. .

Cornil, Mémoire sur le cancer et ses caractères anat., couronné par l'Academie de Paris 12. déc. 1865

Prompt, Bull. de la Soc. anat. 1866. p. 374

Riesenfeld, Diss. Berlin 1868. . . .

Villard, Mouvement méd. Paris 1870

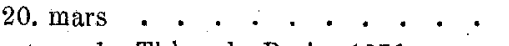

Hess, Diss. Zürich 1872 . . . . . . 11 1 1 - 1 -

Leroux, Bull. de la soe. anat. 1879. p.206 1 - $1-1-$

Mullot, ibidem. 1882. p. 171 . . . . 1 - 1 - 1 -

Plocq, ibidem. 1886. p. 260... . . 1 - $1-1-$

Marchand, D. med. Wochenschr. 1888.

No. 12 . . . . . . . • • • . .

Musser ${ }^{1}$ ), Bost. med. and surg. Journ. 1889.

p. 312.525.553.581... . . . . . 1 - 1 - 1 -

Hans Chiari, Prag. med. Wochenschr.

1890. No. 3

Chachamoviez, Diss. Greifswald $1890.111-1$ - 1 --

H o chenegg, Wien. klin. Wschr. 1890.

No. 3

Morin, Thèse de Paris 1890. . . . . $32{ }^{2} 1-3-$

Peters, Diss. Kiel 1890 . . . . . . $6-6-6-$

Baseler Jahresbericht über die chirurg. Ab-

theilung des Spitals zu Basel. 1890. S.81 1 - 1 - 1 -

Martins, Diss. München 1891 , . . . $2-2-2-$

Thiedemann, Diss. Kiel 1891. . . . 1 - 1 - 1 -

Ohloff, Diss. Greifswald 1891 . . . . . $2{ }^{2} 11_{-}-2$ -

Cumming, Brit. med. Journ. 1891. p. $611-1-1-$

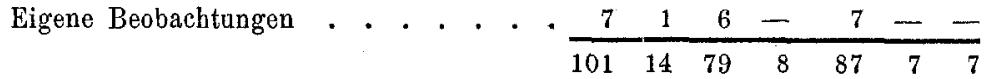

1) Musser's Arbeit konnte ich nicht eingehender berücksichtigen, da mir die Originalarbeiten zum grössten Theil unzugänglich sind. An angeführter Stelle finden sich Einzelfälle nicht erwähnt. 
An hang.

Nicht speciell untersuchte

Fälle:

Klebs, Handbuch der path. Anat. Berlin 1869. S. 492 .

Frerichs, Klinik der Leberkrankheiten.

2. Aufl. S. 45. . . . . . . . . . $11--119-2$

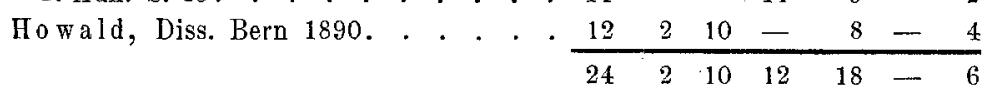

Ehe wir aus dem Resultat dieser Tabelle unsere Schlüsse ziehen, muss ich auf die Fälle H. Zenker's noch besonders eingehen. Er sammelte aus der Literatur 45 Fälle und fügte weitere 8 aus der Erlắnger Sammlung hinzu. Von diesen 53 Fällen betrafen 6 das männliche, 39 das weibliche Geschlecht, $8 \mathrm{Mal}$ vermisst er die Geschlechtsangabe. Gallensteine findet er $41 \mathrm{Mal}$, sie fehlen nach ihm $6 \mathrm{Mal}, 6 \mathrm{Mal}$ ist darüber nichts angegeben. Und zwar findet sich der Galleublasenkrebs ohne Cholelithiasis nach Zenker bei Pepper, Ogle, Haas, Planteau und Kohn. Fraglich erscheint ihm diese bei Moxon und Stiller, bei letzterem nur in einigen Fällen. Nach Einsichtnahme der Originalbeiträge muss ich die Zahlen seiner Tabelle nicht unwesentlich abändern.

Was zunächst den Fall Pepper's anbelangt (American. Journal 1857) so lässt sich derselbe statistisch nicht verwerthen. Er betraf eine Frau von 50 Jahren mit „scirrhöser Entartung" des Uterushalses, des Pankreaskopfes und der Gallenblase. Eine genauere mikroskopische Untersuchung wird nicht mitgetheilt. Ob hier der Pankreaskopf, der in „knorpeligen 'Tumor" verwandelt war, Uterushals oder Gallenblase zuerst erkrankten, bleibt schwer zu entscheiden. Die 3 Fälle $0 \mathrm{gle}$ 's ${ }^{35}$ (J. W. Ogle, St. Georges Hosp. Reports, 1867. Bd. II. S. 346f.) betreffen dessen Beobachtungen No. 17, 18 and 21, und zwar 2 Männer und eine Frau, alle drei mit Gallensteinen. Der fragliche Fall von H. Haas (Prager Vierteljahrsschrift 1876. Bd. 132. S. 136 bis 140) betrifft einen 51jährigen Mann. Bei der Section fanden sich einige Knötchen in der Leber, die Gallengänge dilatirt. Dann sagt das Sectionsprotocoll: „Die beiden Ducti hepatici daumendick erweitert, ebenso der Ductus choledochus. Derselbe ist knapp über dem Eingang des Ductus cysticus bis etwa $2 \mathrm{~cm}$ 
über seiner Müudung in das Duodenum in einen festen, kaum für die Sonde durchgängigen Strang verwandelt, welcher aus fibrösem Gewebe besteht, in das sein Gewebe untergegangen ist. Die Einmündung des Ductus cysticus in den Ductus choledochus kaum bemerkbar. Die Wandungen des ersteren sind ähnlich infiltrirt. Der Fundus der Galleublase in seinen Wandungen von einer $2 \mathrm{~cm}$ dicken, icterisch gefärbten, festen, nur im Centrum erweichten Masse infiltrirt. Pankreas in seinen Zweidrittheilen links atrophisch, sein Kopf an den infiltrirten Ductus choledochus angezogen". Eine specielle Angabe über den Inhalt der Gallenblase fehlt, auch die Möglichkeit, dass der Tumor vom Ductus choledochus ausging, ist vorhanden ${ }^{1}$ ). Primärer Krebs der Gallenwege aber ist eine nur ausnahmsweise mit Cholelithiasis verbundene, besonders beim männlichen Geschlecht beobachtete Affection (Howald a. a. 0.). Der Fall Planteau's betraf eine Frau, die schon früher icterisch gewesen war, aber über etwa vorher bestandene Cholelithiasis nichts anzugeben wusste. Bei der Section war die Gallenblase birnengross, bei verdünnter Wand und eiweissartigem, fadenziehenden Inhalt. Im Fundus fand sich eine Fünffrancsstück grosse, einen Centimeter dicke Geschwulst. Von der Vereinigungsstelle des Ductus cysticus mit dem Ductus hepaticus geht eine Geschwulst aus, welche Arteria hepatica und Vena portarum einschliesst und comprimirt. Ductus choledochus zeigte normale Weite.

Von den 6 Fällen Kohn's ${ }^{3}$ finden sich nach H. Zenker 4 Mal Gallensteine. Nach Kohn's eigenen Angaben finden sie sich in seinen ersten 5 Beobachtungen. Im 6 . Falle fehlten sie allerdings bei der Section, es bestand aber eine GallenblasenColonfistel. Auch hatte der aufgeschnittene Dactus choledochus einen Umfang von $3 \mathrm{~cm}$. Unter solchen Umständen lässt sich ein Schluss betreffs des Vorhandenseins oder Fehlens der Gal-

1) Nach Schluss dieser Mittheilung erhielt ich durch die Güte des Herrn Prof. H. Chiari die schriftliche Bestatigung meiner Ansicht, dass dieser Fall von Has ein primäres Carcinom des Ductus choledochus, nicht der Gallenblase betrifft. Aucb batte Prof. Chiari die Freundlichkeit, mir mitzutheilen, dass er die Richtigkeit meiner Behauptungen an der Hand seines grossen Sectionsmaterials recht oft demonstriren könne. 
lensteine intra vitam mit Sicherheit nicht ziehen. Das Gleiche gilt von Moxon's ${ }^{38}$ Fall, in dem ebenfalls eine - einen Zoll lange - Gallenblasen-Colonfistel beobachtet wurde. Die 5 Fälle Stiller's betreffen 5 Frauen im Alter von $43-59$ Jahren mit Gallensteinen.

Auf Grund dieser Thatsachen ist die Tabelle H. Zenker's folgendermaassen zu ändern: Unter Abzug des Falles Pepper und Haas bleiben 51 Fälle, 43 Frauen und 6 Männer, zweimal fehlt die Geschlechtsangabe. Gallensteine finden sich $48 \mathrm{Mal}$, $2 \mathrm{Mal}$ ist ihre Anwesenheit intra vitam fraglich (Moxon und Kohn), einmal fehlen sie (Planteau).

In den von mir hinzugefügten 48 Fällen finden sich die Gallensteine $46 \mathrm{Mal}$, sie fehlen zweimal bei CorvisartBroca $^{14}$ und Isery ${ }^{16}$. Die Verbältnisse in diesen letzten Fällen sind kurz folgende.

Der Fall Corvisart-Broca betraf eine Frau von 59 Jahren. Die normal grosse Gallenblase war $4-5$ Millimeter dick und hart. Sie enthielt eine klare, serumähnliche Flüssigkeit, etwas blasser und dünnflüssiger als Serum, keine Spur von Galle oder Gallensteinen. Der Ductus choledochus war in einen dicken, derben, undurchgängigen Strang verwandelt, die Gallenblase mündete unvermittelt in einen weiten, dünnwandigen Kanal, den Ductus hepaticus; der Ductus cysticus war erweitert und "verstrichen". Mikroskopisch fanden Broca, Gosselin und Robin ein Carcinom, "scirrboes" in den ältesten Theilen, "encephaloïd" in den Mesenterialdrüsen, dem Netz und den disseminirten Tumoren an der convexen Seite der Leber. Im Falle Icery's war die Gallenblase sehr verdickt, fibrös, und enthielt einen ulcerirten, am Fundus aufsitzenden „Fungus“. Als Inhalt werden schwärzliche stinkende Massen angegeben, das Fehlen von Steinen wird nicht betont. Ductus cysticus sehr verdickt, fast obliterirt bei seiner Mündung in den Ductus choledochus.

Das Resultat der mir bekannten ${ }^{1}$ ), mit Rücksicht auf Chole-

1) Weitere 5 Fälle möchte ich nach Schluss der Arbeit der Vollständigkeit der Literatur wegen noch anführen, ohne dass dadurch das Resultat geändert würde:

Voss, nach Virchow-Hirsch. 1871. Bd.I. S. 160: ein Weib mit Steinen. 
lithiasis genau beschriebenen Fälle, ohne die im Anhang der Tabelle angeführten 24 Beobachtungen ist demnach folgendes: Von 99 Fällen betreffen 14 das männliche, 83 das weibliche Geschlecht, zweimal fehlt die Geschlechtsangabe. Gallensteine finden sich $94 \mathrm{Mal}$, fehlen $3 \mathrm{Mal}$, zweimal lässt sich über ihr Vorhandensein nichts Sicheres nachweisen (Gallenblasen-Colonfistel).

Wie verhalten sich nun die secundären Carcinome der Gallenblase in Bezug auf Cholelithiasis?

In der mir zugänglichen Literatur findet sich keine Abhandlung über diese Frage. Nur zwei Fälle will ich aus der Literatur angeben denen ich 11 weitere aus der hiesigen Sammlung hinzufüge.

1. Riesenfeld (a. a. 0.) Fall 32. Männliches Individuum, Magenkrebs, Metastasen in Pleuren, Peritonäum, Leber, Ductus cysticus und Gallenblase. Keine Gallensteine.

2. van der Regl ${ }^{40}$. Trans. of the path. Soc. London 1875 , B. IX. Männliches Individuum. Pankreaskrebs mit Metastasen in Duct. choledocbus und Gallenblase. Keine Gallensteine.

\section{Eigene Beobachtungen.}

Folgende 11 Fälle von secundärem Krebs der Gallenblase kamen in den letzten 10 Jahren hier zur Section.

1. 1882. No. 207. Gaspard V., 76 Jahre alt. Ulcerirtes Pyloruscarcinom. Metastasen im Peritonäum, der Leber und Gallenblase. Keine Gallensteine.

2. 1883. No. 209. Michel B., 58 Jahre alt. Ulcerirtes Colloidcarcinom der Cardia. Metastasen in Lungen, Pleuren, Zwerchfell, Pankreas, Leber und Gallenblase. Zahlreiche erbsengrosse Tumoren in deren Schleimbaut in's Innere vorragend. Inhalt: blasse, fadenziebende Galle. Keine Gallensteine.

3. 1884. No. 71. Jean A., 67 Jabre alt. Ulcerirtes Pyloruscarcinom. Metastasen in Leber und Gallenblase. Deren Wand verdickt, Schleimbaut höekerig durch Einlagerung von Krebsknoten. Keine Gallensteine.

4. 1884. No. 244. Antoinette Ch., 70 Jahre alt. Ulcerirtes Pylorusearcinom. Metastasen in der Leber, den periportalen Lymphknoten und der Gallenblase. Deren Wand verdickt, markig auf der Schnittfläche, mit vielen kleinen Tumoren durchsetzt. Ductus choledochus comprimirt, Ductus hepa-

Schmidt, Diss. 1891: ein Mann mit Steinen.

Lou is Philipp, Diss. Greifswald 1891: ein Mann mit Gallenblasen-Colonfistel, ohne Steine.

Collinet, Bull, de la Soc. anat. 1892. S. 476 u. 581 : zwei Weiber mit Steinen. 
ticus und cysticus stark erweitert, wie die Gallenblase. Inhalt: viel dünnflüssige Galle. Keine Steine.

5. 1884. No. 246. François M., 65 Jahre alt. Pankreascarcinom. Metastasen in Peritonäum, Leber und Gallenwegen. Ductus choledochus und cysticus verengt, Wandung verdickt, krebsig infiltrirt. Gallenblase sehr verdickt, Schleimhaut enthält knollige, markige Carcinomknoten, die zum Theil ulcerirt sind. Inhalt: flüssiges und geronnenes Blut und schleimige Massen. Kein e Gallensteine.

6. 1885. No. 12. Christian Sch., 62 Jahre alt. Carcinom des Oesophagus. Metastasen in den Lymphknoten längs der Wirbelsäule und im Leberhilus, in der Leber und Gallenblase. Deren Schleimbaut böckerig durch Einlagerung zahlreicher Krebsknoten. Inhalt: fadenziehende, gelbe Galle und einige, kleine Gallensteine.

7. 1885. No. 143. Jean J., 60 Jahre alt. Pankreascarcinom. Metastasen in den retroperitonäalen Lymphknoten, der Leber und Gallenblase. Deren Wand knotig verdickt, Schleimbaut ulcerirt, krebsig infiltrirt. Inhalt: viel gelb-grüne Galle. Keine Gallensteine.

8. 1886. No. 139. Françoise D., 59 Jahre alt. Pankreascarcinom. Metastasen in den Lymphknoten um die Pfortader, in der Leber und Gallenblase. Deren Wand knotig verdickt, Schleimhaut im Bereich des Halses nnd des Ductus cysticus uleerirt, krebsig infiltrirt. Inhalt: schleimige, helle Galle, Detritus der Schleimhaut und ein kirschgrosser Gallenstein.

9. 1887. No. 169. Christophe K., 67 Jahre alt. Pankreascarcinom. Metastasen in Lungen, Pleuren, Milz, Nieren, Leber und Gallenblase. Ductus choledochus durch krebsige Lymphknoten comprimirt. Inhalt der höckerig verdickten Gallenblase: schleimige, helle Galle, keine Gallensteine.

10. 1891. No. 60. Clandine V., 60 Jahre alt. Ulcerirtes Magencarcinom. Metastasen in Peritonäum, Duodenum, Quercolon, Mesenterium, Ovarien und Gallenblase. Deren Mucosa von knotigen Auftreibungen durchsetzt, nicht ulcerirt. Inhalt: schleimige Galle, keine Gallensteine.

11. 1891. No. 199. Andreas W., 52 Jabre alt. Pankreascarcinom. Metastasen in Lungen, Pleuren, Peritonäum, Leber und Gallenblase. Ductus cysticus durch krebsige Lymphknoten comprimirt. Mehrere erbsengrosse Krebsknoten in der Schleimhaut der Gallenblase. Inhalt: viele schleimige, sehr eingedickte Galle, keine Gallensteine.

Von diesen 13 Fällen kommen demnach 10 auf das männliche, 3 auf das weibliche Geschlecht. Gallensteine finden sich zweimal, ihr Fehlen wird ausdrücklich bemerkt 11 Mal.

Welch ein Unterschied zwischen primärem und secundärem Krebs der Gallenblase in Bezug auf Cholelithiasis und Geschlecht! Zur besseren Uebersicht stellen wir das Resultat beider Untersuchungen neben einander und zwar in Procenten ausgedrückt. 


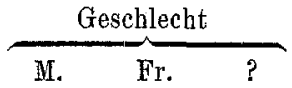

Prim. Gallen-

blasenkrebs:

Secund. Gallen-

blasenkrebs:
15 pot. 83 pCt. 2 pCt.

77 pCt. 23 pCt. - pCt.

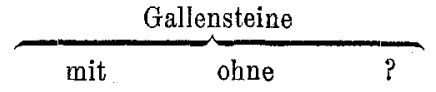

$95 \mathrm{pCt}$.

Durch diese Thatsachen ist mit Sicherheit bewiesen, dass Krebs der Gallenblase an und für sich nicht mit Nothwendigkeit Cholelithiasis bedingt. Kommt solche beim secundären Krebs ausnahmsweise einmal vor, so kann sie ebensowohl vorher bestanden, als sich während dessen gebildet haben.

Selbst in vorgerückten Stadien des Zerfalls des Gallenblasenkrebses, soweit er von Gallensteinen nicht bedingt ist, sehen wir dieselben fehlen. Dies entspricht auch vollkommen den gültigen Anschauungen über die Bildung der Concremente im Allgemeinen. Die Bedingungen für ihre Entstehung sind nach von Recklinghausen ${ }^{41}$ dreierlei Art: 1. dass das sie bildende Material in der betreffenden Flüssigkeit im Ueberschuss vorhanden ist, 2. dass diese in ihrer normalen Entleerung aus irgend welcher Ursache verzögert wird, 3. dass ein solider Fremdkörper oder irgend eine feste Masse den Kern abgiebt, auf den ihre Ausscheidung aus der Flässigkeit erfolgt. Alle diese Bedingungen werden durch die Entstehung eines Carcinoms in der Gallenblasenwand nicht in jedem Falle erfüllt, wohl aber kann ein Krebs, der dieselbe in grosser Ausdehnung infiltrirt, dadurch eine Behinderung ihrer Contraction und eine annormale Zusammensetzung des Inhaltes verursachen. Kommt dann die Ulceration der Geschwulst hinzu, so kann eine Abscheidung auf deren nekrotische Partikel allerdings erfolgen. Andererseits folgt aus dem Umstande, dass beim primären Krebs der Gallenblase fast ausnahmslos Cholelithiasis beobachtet wird, und zwar selbst in Fällen, in denen makroskopisch eine Veränderung der Gallenblase nicht nachzuwiesen ist, - Fall 7 eigener Beobachtung -, dass die Gallensteine in der Aetiologie die bei weitem erste Rolle spielen. Und auch dieser Schluss findet sich in schönstem Einklang mit der Lehre von den Geschwülsten im Allgemeinen. Dass in deren Aetiologie dem mechanischen Reize, sei es dass er acut oder chronisch einwirkt, die grösste Bedeu- 
tung zukommt, ist eine bekannte Thatsache. Gerade bei der Frau, wo Gallensteine so viel häufiger vorkommen als beim Manne und wo in dem Schnüren ein weiterer "mechanischer Reiz" gegeben ist, sehen wir den primären Krebs der Gallenblase 5-6 Mal häufiger als beim Manne. Der secundäre hingegen findet sich im Gegentheil überwiegend beim männlichen Geschlecht, entsprechend der Thatsache, dass die ihn veranlassenden primären Geschwülste, besonders des Digestionstractus, beim Manne häufiger sind, als beim Weibe. Allerdings beweisen für den secundären Gallenblasenkrebs 13 Fälle nicht viel, und eine Aufgabe der Statistik wird es sein, durch Zusammenstellung neuer Fälle diesen Schlüssen eine breitere Basis zu geben.

Sicher aber erlauben die aus dem Vergleich des primären Gallenblasenkrebses mit dem secundären sich ergebenden Thatsachen folgende Schlüsse:

1. Gallensteine finden sich beim primären Krebs der Gallenblase fast ausnahmslos, beim secundären nur ausnahmsweise.

2. Sie sind jedenfalls eine der Ursachen des Gallenblasenkrebses, sicher nicht die Folge desselben.

$$
\text { L i t e ra t u r. }
$$

1. Bertrand, Thèse de Paris. 1870.

2. Behrens, Dissert. Strassburg 1879.

3. Kohn, Diss. Breslau 1879.

4. Kraus, Diss. Leipzig 1884.

5. Zenker, Diss. Erlangen 1889.

6. Chachamovicz, Diss. Greifswald 1890.

7. Morin, Thèse de Paris. 1890.

8. Peters, Diss. Kiel 1890.

9. Martins, Diss. Kiel 1891.

10. Thi edemann, Diss. München 1891.

11. Ohloff, Diss. Greifswald 1891.

12. Klebs, Handbuch der path. Anatomie. Berlin 1869. S. 492.

13. Lacaze-Duthiers, Bulletin de la Société anatomique. 1847. p. 335.

14. Corvisart-Broca, ebenda. 1848. p. 41 etc.

15. Rippol, ebenda. 1849. p. 359.

16. Icery, ebenda. 1853. p. 73.

17. Mabieux, ebenda. 1853. p. 180.

18. Cornil, Sur le cancer et ses caractères anatom. Paris 1865. 12. déc. 
19. Prompt, Bullet. de la Soc. anat. 1866. p. 374 .

20. Riesenfeld, Diss. Berlin 1868.

21. Villard, Mouvement médical. Paris 1870. 20 mars.

22. Hess, Diss. Zürich 1872 .

23. Leroux, Bullet. de la Soc. anat. 1879. p. 206 .

24. Mullot, ibid. 188\%. p. 171.

25. Plocq, ibid. 1886. p. 260 .

26. Marchand, Deutsebe med. Wochenschrift. 1890. No. 3.

27. Muster, Boston med. and surg. Journal. 1889. p. 525. 553. 581.

28. Chiari, Prag. med. Wochenschrift. 1890. No. 3.

29. Hochenegg, Wiener klin. Wochenschrift. 1890. No. 3.

30. Basler Jahresbericht über die chir. Abth. des Spit. zu Basel. 1890. S.81.

31. Cumming, Brit. med. Journal. 1891, p. 61.

32. Frerichs, Klin. der Leberkrankbeiten. 2. Aufl. S. 45.

33. Howald, Diss. Bern 1890.

34. Pepper, American. Journ. 1857. Schmidt's Jahrb. Bd. 95. S. 322.

35. Ogle, St. Georges Hosp. Reports 1867. Bd. II. S. 346 u. ff.

36. Haas, Prager Vierteljahrschrift. 1876. Bd. 132. S. 136-140.

37. Planteau, Bull. de la Soc. anat. 1875. p. 689.

38. Nox on, Trans. of the path. soc. 1868. T. 18. p. 140.

39. Stiller, Pesth. med. chir. Presse. 1886. No. 2. Centralbl. für klin.

Med. 1887, p. 227.

40. van der Reyl, Trans. of the patb. Society. London 1875. T. 9.

41. von Recklinghausen, Handbuch der allg. Path. Stutgart 1883. S. 390 .

\section{XVII. \\ Kleinere Mittheilungen.}

1. Notiz über das Verhalten der Pentaglykosen (Pentosen)
im menschlichen Organismus.

Von Wilhelm Ebstein in Göttingen.

Herr M. Cremer hat mir die „Ergebnisse seiner Fütterungsversuche mit Pentosen"1) zugeschickt, worin es heisst, dass meine Ansicht, dass

1) Sitzungsberichte der Gesellschaft für Morphologie und Physiologie zu München. 1893. Heft 1. Sonderabdruck. 